\title{
Grupos de convivência como suporte na prevenção da depressão em idosos
}

\author{
Coexistence groups as a support for the prevention of \\ the depression in the elderly
}

\begin{abstract}
Andreza Guimarães de Oliveira', Sanmille Santos Santiago de Abreu' ${ }^{2}$ Maria Aparecida Silva Santos Macedo ${ }^{3}$, Stênio Fernando Pimentel Duarte ${ }^{4}$, Luciana Araújo dos Reis ${ }^{5}$, Pollyanna Viana Lima ${ }^{6}$

'Faculdade Independente do Nordeste - FAINOR, Vitória da Conquista, Bahia, Brasil. andreza.guimaraes@outlook.com ${ }^{2}$ Faculdade Independente do Nordeste - FAINOR, Vitória da Conquista, Bahia, Brasil.mille_sba@hotmail.com ${ }^{3}$ Faculdade Independente do Nordeste - FAINOR, Vitória da Conquista, Bahia, Brasil. lyasoyer@hotmail.com ${ }^{4}$ Faculdade Independente do Nordeste - FAINOR, Vitória da Conquista, Bahia, Brasil. sfernando@fainor.com.br ${ }^{5}$ Universidade Estadual do Sudoeste da Bahia - UESB/Faculdade Independente do Nordeste - FAINOR, Vitória da Conquista, Bahia, Brasil. lucianauesb@yahoo.com.br ${ }^{6}$ Autora para correspondência. Universidade Estadual do Sudoeste da Bahia - UESB/Faculdade Independente do Nordeste - FAINOR, Vitória da Conquista, Bahia, Brasil. polly_vl@yahoo.com.br
\end{abstract}

\begin{abstract}
RESUMO | OBJETIVO: Avaliar sinais de depressão em idosos participantes e não participantes de grupo de convivência. METODOLOGIA: Trata-se de um estudo do tipo exploratório, com abordagem quantitativa, que ocorreu em um município da Região Sudoeste da Bahia. Foram avaliados 18 idosos que frequentam grupos de convivência e 18 que não frequentam, totalizando 36 idosos. Os instrumentos utilizados foram Mini Exame do Estado Mental, Questionário socioeconômico e escala de Depressão de Beck. RESULTADOS: Dos 36 idosos avaliados, a maioria era do sexo feminino $(88,9 \%)$, que vivem sozinhos e são viúvos, solteiros ou divorciados $(83,3 \%)$ e de classe predominante $\mathrm{E}(77,8 \%)$. Verificou-se que $33,3 \%$ dos idosos que frequentam grupos de convivência apresentaram depressão, já os idosos que não frequentam totalizaram 50\%. CONCLUSÃO: Os grupos mostraram-se relevantes, pois podem ser considerados preventivos, proporcionando aos idosos uma melhor interação social e enfrentamento do processo de envelhecimento.
\end{abstract}

DESCRITORES: Idosos. Envelhecimento. Depressão. Socialização. Saúde mental.

\begin{abstract}
I OBJECTIVE: To evaluate depression in elderly participants and non-participants in a coexistence group. METHODOLOGY: This is an exploratory study, with a quantitative approach, that occurred in a municipality in the Southwest Region of Bahia. We evaluated 18 elderly people who attend groups of coexistence and 18 who do not attend, totaling 36 elderly. The instruments used were the Mental State Mini Exam, the Socioeconomic Questionnaire and the Beck Depression Scale. RESULTS: Of the 36 elderly, the majority were female $(88.9 \%)$, who live alone and are widowed, single or divorced (83.3\%) and predominant class E $(77.8 \%)$. It was verified that $33.3 \%$ of the elderly that attend groups of coexistence presented depression, whereas the elderly that do not attend totaled 50\%. CONCLUSION: The groups were relevant because they can be considered preventive, providing the elderly with a better social interaction and coping with the aging process.
\end{abstract}

DESCRIPTORS: Elderly. Aging. Depression. Socialization. Mental health. 


\section{Introdução}

O Brasil encontra-se em mudanças demográficas significativas, representadas pela queda da taxa de mortalidade, seguida pela queda da taxa de natalidade. Isto pode estar relacionada a diminuição da taxa de fecundidade média da mulher brasileira, atrelada a redução da taxa de mortalidade $^{2}$, a melhoria do padrão de vida populacional, maior acesso a inovação médica, programas de saúde, além dos cuidados pessoais e saneamento básico', aumentando assim a esperança e dobrando o tempo médio de vida da população brasileira.

A população idosa é alvo dos olhares de áreas distintas do conhecimento, por estar sendo a de maior crescimento ${ }^{3}$. Estima-se que em 2040 haverá 153 idosos para cada 100 jovens ${ }^{4}$, mantendo a perspectiva de crescimento até 2050, o que assegura um novo tempo em que a população idosa cresce e finca raízes. ${ }^{5}$ Envelhecer é um processo natural, porém complexo, traz inúmeros desafios, envolve diversos aspectos, alterações biopsicossociais, perdas, dependência, privações e todo o oposto do que o ser humano almeja e vive até então, tendo pois associação direta com o histórico do indivíduo, seu contexto cultural, sua aceitaçãob.

Vale lembrar que essas mudanças podem tornar os idosos fragilizados e dependentes, provocar sentimentos negativos, principalmente devido a perda de autonomia, a vulnerabilidade e, sobretudo, tornar algumas pessoas susceptíveis a agravos de problemas de saúde e até aparecimento de doenças, 6 como a depressão.

A depressão entendida como um transtorno do humor, perda da capacidade de sentir interesse em tudo, melancolia e queda da autoestima, tem sido destaque na população idosa ${ }^{7}$ e é considerada um problema de saúde relevante ${ }^{8}$. Suas características são devastadoras, destroem a graça pelo viver, a esperança de dias melhores e trazem consigo um turbilhão de sentimentos negativos, destruindo autoestima, fazendo da tristeza o seu combustível e os quadros mais graves de depressão podem ter como desfecho o suicídio ${ }^{7}$. Porém, no idoso nem sempre as características são claras, dificultando o diagnóstico, que pode ser confundido devido às mudanças físicas e neurológicas associadas ao processo de enveIhecimento ${ }^{8}$.
No ajustamento pessoal ao processo de envelhecimento, o indivíduo pode tornar-se vulnerável a depressão, isso porque os idosos sofrem algumas privações e até exclusões, perdem espaço no mercado de trabalho, consequentemente ganham menos, e dessa maneira sobrevivem em uma sociedade, que na maioria das vezes dita o seu futuro e restrições, que troca oportunidade por ajudas caridosas ${ }^{8}$.

Acrescenta-se a isso o fato de o processo de envelhecimento envolver medo da inutilidade, dependência e abandono, isolamento, exclusão, vulnerabilidade, rompimento de laços, o que pode provocar sinais de tristeza e sentimentos negativos ${ }^{9}$.

Estudos têm descrito a importância de grupos de convivência para idosos, especialmente no que se refere aos benefícios para a proteção do idoso e para $\circ$ distanciamento de pensamentos e atitudes negativas. Isso porque estes grupos são locais que contribuem positivamente para a população idosa, no sentido que favorece aos mesmos desempenharem atividades sociais, exercerem suas habilidades e estabelecerem vínculos de amizade. Além do mais, esses encontros possibilitam momentos prazerosos e contribuem na restauração da auto-estima ${ }^{10,11}$.

Diante do exposto, é possível afirmar que a temática ora proposta é relevante, pois a sociedade continuará envelhecendo ${ }^{8}$ e $\circ$ aumento de idosos com depressão tende a elevar devido à fragilidade desses indivíduos ao longo do tempo ${ }^{12}$, situação que requer o conhecimento do processo de envelhecimento, dos sinais de depressão, para permitir a compreensão dos profissionais e familiares frente aos novos desafios.

Além disso, este estudo justifica-se pelo fato de discutir um tema de relevância para a saúde públi$\mathrm{ca}$, que que tem impactado na qualidade de vida de muitos brasileiros. Para tanto, tem-se a seguinte questão de pesquisa: os grupos de convivência são importantes para o suporte na prevenção de depressão?

Para responder a esse questionamento, tem-se como objetivo avaliar os sinais de depressão em idosos participantes e não participantes de grupo de convivência. 


\section{Método}

Trata-se de um estudo do tipo exploratório, com abordagem quantitativa, que avaliou os sinais de depressão em 36 idosos de 60 anos ou mais, de ambos os sexos. Sendo 18 idosos que participam de grupos de convivência e 18 que não participam.

A pesquisa ocorreu em um grupo de convivência e em uma unidade básica de saúde sem grupo de convivência, localizados em um município da região Sudoeste da Bahia, Brasil, durante os meses de julho a dezembro de 2016. A escolha do grupo de idosos dessas unidades se deu pelo fator acessibilidade e devido a unidade básica ser conveniada com a instituição onde os pesquisadores estudam e trabalham.

grupo de convivência tinha em média 30 idosos participantes e na unidade básica, 50 idosos. Porém nos dois locais de pesquisa, nem todos se enquadravam aos critérios de inclusão da pesquisa, alguns se recusaram a participar e outros não estavam no local quando foi realizada a coleta de dados, assim participaram do estudo 36 idosos. Ressalta-se que a quantidade exata, 18 idosos em cada local/grupo foi escolha dos pesquisadores para que a comparação fosse mais fidedigna.

Os critérios de inclusão para a participação dos idosos foi: ter 60 anos ou mais; participar do grupo de convivência escolhido para a pesquisa ou estar cadastrado na unidade básica selecionada; de qualquer sexo, renda ou religião; e ter cognitivo preservado, avaliado pelo Mini Exame do Estado Mental. Já os critérios de exclusão foi idosos acamados.

Para a coleta de dados foram utilizados os seguintes instrumentos: Mini Exame do Estado Mental (MEEM) ${ }^{13}$; Escala de Depressão de Beck $^{14}$ e o Questionário socioeconômico. O MEEM foi utilizado como condição para comprovação da capacidade de cognição e, portanto para incluir na pesquisa os idosos com capacidade de responder ao questionário. Ele é constituído por 10 questões e avalia quatro critérios: memória a curto e longo prazo; orientação; informações cotidianas e capacidade de calcular. Sua pontuação é calculada e estabelecida segundo as funções cognitivas mantidas, sendo pontuada entre 30 a 26 pontos, não sugestiva de déficit entre 26 a 24 pontos e 23 pontos ou menos sugestivo de déficit cognitivo ${ }^{13}$.
- A escala de depressão - trata de um auto-relato, sendo importante na classificação da intensidade. Esta escala é composta por 21 itens: tristeza; pessimismo; frustração; desgosto; culpabilidade; tortura; sentimentos negativos por si; sentimento de culpa; vontade de cometer suicídio; lamentação; irritabilidade; solidão; insegurança; baixa autoestima; dificuldade de concentração; insônia; fadiga; alteração no apetite; emagrecimento; aflição quanto ao estado de saúde e frigidez. Cada item possui um valor de intensidade avaliado entre 0 a 3 pontos e a soma classifica-se segundo o grau de depressão ou ausência da doença. Nesse sentido, a pontuação 10 é definida como "sem depressão ou depressão leve", 10 a 18 pontuados como "grau de depressão leve a moderado", 19 a 29 pontos como "grau de depressão moderada a grave" 14 .

- O Questionário socioeconômico foi composto por perguntas referentes a alguns aspectos sociais e econômico como: renda, a contribuição pessoal de cada um e a quantidade de pessoas sustentadas com essa renda; avaliação da classe; estado civil e o sexo.

Os idosos responderam aos questionários com auxílio de entrevistadores previamente treinados, constituído por discentes participantes do grupo de pesquisas, bem como professores/pesquisadores, que também são integrantes do grupo de pesquisa, em locais reservados, ventilados, sem barulho, o que possibilitou ao idoso responder sem interrupções e/ ou indução das respostas. Os questionários foram digitais, as respostas foram gravadas e enviadas ao Google drive após a finalização.

Para a análise estatística houve a utilização do programa estatístico Statistical Package For The Social Sciences (SPSS), 23.0, tendo $<0,05$ como nível de significância.

Este estudo está inscrito em uma pesquisa do Núcleo de Extensão, Pesquisa e Estudo de Doenças Crônicas, que tem o objetivo de identificar o perfil epidemiológico das doenças crônicas na Região Sudoeste da Bahia. $O$ presente estudo seguiu os preceitos éticos, sendo submetido à aprovação do comitê de ética em pesquisa da Faculdade Independente do Nordeste com Certificado de Apresentação para Apreciação Ética (CAAE) $n^{\circ}$ 
448762.15.8.0000.5578 e parecer de aprovação $\mathrm{n}^{\circ}$ 1.859.545. Os idosos foram devidamente orientados e esclarecidos sobre o objetivo da pesquisa, bem como sobre o Termo de Consentimento Livre e Esclarecido, o qual foi assinado pelos idosos, e somente depois das informações e assinaturas, os procedimentos da coleta foram iniciados.

\section{Resultados}

A Tabela 1, referente à distribuição dos idosos que frequentam e que não frequentam Grupos de Convivência, de acordo com as variáveis sociodemográficas e econômicas, mostra que os idosos que participavam de grupos de convivência tinham entre 60 e 80 anos, com média de 71,9. E os que não participavam tinham entre 60 e 89 anos, com média de 69,6 .

No que se refere ao sexo, tanto os idosos que frequentam grupos de convivência, quanto os que não frequentam são na sua maioria do sexo feminino, respectivamente, $88,9 \%(16)$ e $77,8 \%$ (14).

Quanto ao estado civil dos idosos que participavam de grupos, $16,7 \%$ são casados (3) e $83,3 \%$ (15) são viúvos, divorciados ou solteiros. Dos idosos que não participavam de grupos $38,9 \%$ (7) são casados e $61,1 \%$ são viúvos, divorciados ou solteiros.

Em relação a classe social dos idosos que participavam de grupos de convivência, 5,5\% (1) pertencem a classe C, $16,7 \%$ (3) a classe $D$ e $77,8 \%$ (14) a classe E. Já os idosos que não participam de grupos, 5,6 (1) são pertencentes a classe $C, 0 \%$ a classe $D$ e $94,4 \%$ pertencentes a classe $E$.

Em relação às atividades laborais, 5,6\% (1) dos idosos que frequentam grupos de convivência afirmaram que trabalham e 94,4\% (17) que não trabalham. Já os idosos que não participavam de grupos $22,2 \%$ (4) afirmaram que trabalham e $77,8 \%$ (14) que não trabalham.

Tabela 1. Distribuição dos idosos que frequentam e que não frequentam Grupos de Convivência, de acordo com as variáveis sociodemográficas e econômicas. Vitória da Conquista - BA, 2017

\begin{tabular}{cccccc} 
& & & \multicolumn{2}{c}{ Idosos que frequentam } & \multicolumn{2}{c}{ Idosos que não frequentam } \\
& Variáveis & grupos de convivência & \multicolumn{2}{c}{ grupos de convivência } \\
& & $\mathbf{N}$ & $\%$ & $\mathbf{N}$ & $\%$ \\
\hline Sexo & Feminino & 16 & 88,9 & 14 & 77,8 \\
& Masculino & 2 & 11,1 & 4 & 22,2 \\
Estado & Casado & 3 & 16,7 & 7 & 38,9 \\
Civil & Outros & 15 & 83,3 & 11 & 61,1 \\
& Classe C & 1 & 5,5 & 1 & 5,6 \\
Classe' & Classe D & 3 & 16,7 & 0 & 0 \\
Social & Classe E & 14 & 77,8 & 17 & 94,4 \\
& Sim & 1 & 5,6 & 4 & 22,2 \\
Trabalho & Não & 17 & 94,4 & 14 & 77,8
\end{tabular}

' Segundo o Centro de Políticas a Classe $E$ recebe entre $R \$ 0,0$ até $R \$ 1.254$, a classe $D$ de $R \$ 1.255$ até $R \$ 2.004$ e a classe C R\$2.005 a R\$ 8.640. Disponivel em: https://cps.fgv.br/qual-faixa-de-rendafamiliar-das-classes 
A Tabela 2 apresenta a distribuição dos idosos que frequentam e que não frequentam Grupos de Convivência acerca da depressão, avaliados pela Escala de Depressão de Beck. Esta é uma escala efetiva para avaliação de sintomas característicos da depressão, porém vale ressaltar que ela sozinha não pode mensurar que um indivíduo tem depressão, mas possibilita perceber indícios para que seja investigada mais profundamente. Verificou-se que $66,7 \%$ (12) dos idosos que frequentam grupos de convivência não apresentaram sinais de depressão e 33,3\% (6) apresentaram. Já os idosos que não frequentam grupos, 50\% (9) apresentaram sinais da doença e $50 \%$ (9) não apresentaram.

Tabela 2. Distribuição dos idosos que frequentam e que não frequentam Grupos de Convivência, de acordo com a variável depressão. Vitória da Conquista - BA, 2017

Idosos que frequentam grupos de Idosos que não frequentam grupos de

\begin{tabular}{cccccc}
\multirow{2}{*}{ Variáveis } & \multicolumn{2}{c}{ convivência } & \multicolumn{2}{c}{ convivência } \\
\cline { 3 - 6 } & & $\mathbf{N}$ & $\%$ & $\mathbf{N}$ & $\%$ \\
\hline \multirow{2}{*}{ Sem } & 12 & 66,7 & 9 & 50 \\
\hline
\end{tabular}

Fonte: Os autores (2019).

\section{Discussão}

processo de envelhecimento pode ser marcado por desânimo, isolamento, doenças, desmerecimento, entre outros pontos negativos, pois erroneamente, os idosos ainda são vistos como inúteis pela sociedade, e sofrem pelo desprezo ${ }^{11}$.

Os grupos de convivência são fundamentais na inclusão dos idosos na sociedade ${ }^{15}$ e o envolvimento nestes grupos é positivo, pois os idosos lidam com pessoas da mesma faixa etária, dividem seus sentimentos (sejam eles positivos ou negativos), seus conhecimentos, perdas, reduzem sentimentos como angústia e insegurança, oferecem e recebem afago, compartilham momentos e saberes da vida, além de motivação e apoio emocional ${ }^{16}$. O que favorece na prevenção da depressão ou mesmo serve como apoio aqueles que já encontram-se neste quadro.

A depressão aumenta a morbimortalidade, provoca impactos na funcionalidade e na qualidade de vida, portanto, deve ser acompanhada pelos profissionais de saúde no sentido de reduzir os sintomas consequentes da doença e tratar por completo para evitar recidiva ${ }^{12}$.

O presente estudo evidenciou que os idosos que participam dos grupos de convivência têm a média de idade de 71,9 anos, não tendo semelhança direta com outros estudos ${ }^{17-19}$ que apresentaram, res- pectivamente, a média de 70,$1 ; 72,03$; e 65 anos. Porém, percebe-se que estes resultados se aproximam.

Os participantes de grupos de convivência são predominantemente do sexo feminino, semelhante a outro estudo que aponta que o perfil dos participantes brasileiros em grupos de convivência é, em sua maioria, composto por mulheres, os homens participantes são poucos e na maior parte são acompanhantes de suas esposas ${ }^{16}$. Outro estudo também observou uma menor participação dos homens nesses encontros e chegou a conclusão de que eles mostram-se mais resistentes, muitas vezes por preconceitos ou mesmo por medo de serem caracterizados como frágeis ${ }^{11}$.

Os idosos participantes são, em sua maioria, viúvos, divorciados ou solteiros. Esse perfil pode ser justificado pela menor longevidade do homem, com a preferência da mulher em ficar sozinha, mantendo as lembranças do ex-parceiro, muitas vezes até o final da vida e a escolha dos homens idosos por mulheres mais jovens ${ }^{5}$.

A maioria dos idosos participantes era de baixa renda e escolaridade, assemelhando-se aos resultados dos estudos de Miranda, Mendes e Silva; Alves $^{5,1}$. No que se refere à atividade laboral, os idosos, em sua maioria, não a realizam e são aposentados. Dado semelhante ao encontrado na pesquisa de Andrade et al. ${ }^{11}$. O apoio social é de suma 
importância para pessoas idosas de baixa renda e para quem parou de trabalhar e se aposentou, ele aumenta a capacidade de sobrevivência em situações inesperadas e melhora significativamente a qualidade de vida.

Concernente à depressão, o estudo mostrou um menor índice da doença nos idosos que se integram em grupos de convivência, sendo estes grupos de grande relevância no processo de envelhecimento, pois possibilitam a ressocialização, visto que os idosos têm uma vida ativa, praticam atividades físicas, exploram seus novos limites, compartilham saberes e consequentemente ganham uma melhora na qualidade de vida.

No decorrer do tempo foram mantidos preconceitos em relação aos idosos, associando a eles decadência e privações, impedindo uma visão ampla dessa fase ${ }^{20}$. Por outro lado, os grupos surgiram para contribuírem na identificação do idoso frente ao envelhecimento e enfrentamento de seus receios, possibilitando que os idosos descubram os bônus dessa fase, e que a percebam como um recomeço e não um fim, sendo imprescindível a convivência em grupo, pois eles contribuem na melhora da saúde e equilíbrio emocional através dos vínculos afetivos, que são fundamentais para a integração a uma rede social ${ }^{16}$. Ademais, os grupos proporcionam relações e atividades que causam satisfação nos idosos, especialmente em relação a hábitos saudáveis, favorecendo melhora significativa na qualidade de vida ${ }^{16}$.

Quanto aos idosos que não participam de grupos, os resultados foram similares no que se refere à depressão, visto que demonstrou que metade dos idosos apresentou depressão e a outra metade não, - que aponta a necessidade de observação, pois esses que não apresentaram a doença podem estar vulneráveis diante a possibilidade do desencadeamento de sentimentos negativos, devido às mudanças do processo de envelhecimento, que podem causar estranheza.

A depressão e o envelhecimento têm intensa relação com a diminuição de atividades rotineiras e da saúde, possível dependência, entre outros fatores, demonstrando a necessidade de identificação correta sobre si e a nova fase ${ }^{21}$. Nesse sentido, é de extrema importância a convivência em grupo, pois possibilita ao idoso dividir os seus anseios, aprender a lidar com as novas mudanças, ser amparado, além de ser influenciado a ter hábitos para um melhor padrão de vida, e consequentemente de uma melhor saúde, tanto física, mas principalmente a mental ${ }^{1}$. Além disso, contribui de forma efetiva no enfrentamento aos desafios gerados por essa nova fase e no afastamento dos fatores de risco da doença, como a solidão e o isolamento social.

Esse estudo teve certa limitação devido a quantidade de idosos que se dispuseram de forma livre a participar, mas o seu objetivo foi alcançado. $O$ estudo demonstrou uma menor quantidade de idosos com depressão nos grupos de convivência, identificando a influência positiva destes grupos no processo de envelhecimento frente a depressão.

\section{Conclusão}

Avaliar a depressão em idosos que participam e idosos que não participam de grupos de convivência foi uma possibilidade de colocar em voga um tema de extrema relevância. Os achados apontam os benefícios dos grupos de convivência, que podem ser considerados preventivos, pois proporcionam atividades, viagens, eventos, bate-papo entre outros., e é algo que insere o idoso novamente na sociedade, porém nem todos têm acesso aos seus benefícios, muitas vezes pela dificuldade de locomoção ou até mesmo devido a sentimentos negativos, principalmente de solidão e exclusão social.

Diante disso, torna-se relevante a necessidade do profissional se aprofundar em conhecer os ônus e bônus do envelhecimento para ajudar o idoso a triIhar um envelhecimento digno e ativo, ainda que isso comece nas unidades de saúde, devido a facilidade, de acordo com o acompanhamento dos profissionais de cada unidade.

O processo de envelhecimento é amplo e rico, não sendo apenas um lugar a chegar, mas um caminho a percorrer. Infelizmente, de forma errônea, o envelhecer é associado a fraqueza e adoecimento. Uma visão inadequada, pois o envelhecimento pode ocorrer de maneira saudável e os grupos contribuem para isso, possibilitando ao idoso uma saúde mental, física e social. 
Frente ao exposto, aponta-se a necessidade de mudanças de hábitos, adaptação, aceitação e principalmente a quebra de conceitos e associações errôneas ligadas ao envelhecimento. Salienta-se, portanto, a necessidade cada vez mais urgente de investimento em grupos de convivência que dêem possibilidade aos idosos de uma integração ativa nos meios sociais, com manutenção da saúde mental e consequentemente uma melhora da qualidade de vida.

\section{Conflitos de interesses}

Nenhum conflito financeiro, legal ou político envolvendo terceiros (governo, empresas e fundações privadas, etc.) foi declarado para nenhum aspecto do trabalho submetido (incluindo mas não limitandose a subvenções e financiamentos, conselho consultivo, desenho de estudo, preparação de manuscrito, análise estatística, etc.).

\section{Referências}

1. Alves JED. Transição demográfica, transição da estrutura etária e envelhecimento. Revista Portal de Divulgação. $2014 ; 40(4): 8-15$.

2. Mello MV. O envelhecimento da população brasileira: intensidade, feminização e dependência. Rev Bras Estudos de Pop. $1998 ; 15(1)$.

3. Dawalibi NW, Anacleto GMC, Witter C, Goulart RMM, Aquino RC. Envelhecimento e qualidade de vida: análise da produção científica da SciELO. Estud psicol. 2013;30(3):393403. doi: $10.1590 /$ S0103-166X2013000300009

4. Miranda GMD, Mendes ACG, Silva ALA. O envelhecimento populacional brasileiro: desafios e consequências sociais atuais e futuras. Rev Bras Geriatr Gerontol. 2016;19(3):507519. doi: $10.1590 / 1809-98232016019.150140$

5. Silva JVF, Silva EC, Rodrigues APRA, Miyazawa AP. A relação entre o envelhecimento populacional e as doenças crônicas não transmissíveis: sério desafio de saúde pública. Ciências Biológicas e da Saúde. 2015;2(3):91-100.

6. Gomes JB, Reis LA. Descrição dos sintomas de Ansiedade e Depressão em idosos institucionalizados no interior da Bahia, Brasil. Kairós Gerontologia. 2016;19(1):175-191.

7. Abelha L. Depressão, uma questão de saúde pública. Cad Saúde Colet. 2014;22(3):223. doi: 10.1590/1414$\underline{462 \times 201400030001}$
8. Martins RM. A depressão no idoso. Millenium-Journal of Education, Technologies, and Health 2016;34(13):1 19-123.

9. Sommavilla L. O sofrimento psíquico dos idosos [monografia]. ljuí: Universidade Regional do Noroeste do Estado do Rio Grande do Sul; 2015.

10. Soares SMS, Coronago VMMO. Grupos de Convivência: Influência na Qualidade de Vida da Pessoa Idosa. Id on Line Rev Psic. 2016;10(33):1 27-140. doi: 10.14295/idonline. $\underline{\mathrm{v} 10 i 33.603}$

11. Andrade NA, Nascimento MMP, Oliveira MMD, Queiroga RM, Fonseca FLA, Lacerda SNB et al. Percepção de idosos sobre grupo de convivência: estudo na cidade de CajazeirasPB. Rev Bras Geriatr Gerontol. 2014;17(1):39-48. doi: $10.1590 /$ S1809-98232014000100005

12. Lima AMP, Ramos JLS, Bezerra IMP, Rocha RPB, Batista HMT, Pinheiro WR. Depressão em idosos: uma revisão sistemática da literatura. R Epidemiol Control Infec. 2016;6(2):97-103. doi: $10.17058 /$ reci.v6i2.6427

13. Folstein MF, Folstein SE, Mchugh PR. "Mini-mental state": a practical method for grading the cognitive state of patients for the clinician. J Psychiatr Res. 1975;12(3):189-198.

14. Beck ATMD, Ward MDCH, Mendelson MDM, Mock MDJ, Erbaugh MDJ. An inventory for measuring depression. Arch Gen Psychiatry. 1961;4(6):561-571.

15. Dalmolin IS, Leite MT, Hildebrandt LM, Sassi MM, Perdonssini LGB. A importância dos grupos de convivência como instrumento para a inserção social de idosos. Revista Contexto \& Saúde. 2011 ; 10(20):595-598. doi: $10.21527 / 2176-7114.2011 .20 .595-598$

16. Wichmann FMA, Couto AN, Areosa SVC, Montañés MCM. Grupos de convivência como suporte ao idoso na melhoria da saúde. Revista Brasileira de Geriatria e Gerontologia. 2013;16(4):821-832. doi: 10.1590/S1809$\underline{98232013000400016}$

17. Lustosa LP, Marra TA, Pessanha FPAS, Freitas JC, Guedes RC. Fragilidade e funcionalidade entre idosos frequentadores de grupos de convivência em Belo Horizonte, MG. Rev Bras Geriatr Gerontol. 2013;16(2):347-354. doi: 10.1590/ $\underline{\mathrm{S} 1809-98232013000200014}$

18. Cruz RVS, Pitanga CDS, Gonçalves BO, Moura MVA, Gonzaga PD. Avaliação do risco de violência contra idosos participantes de um grupo de convivência em ltabuna, BA. Memorialidade. 2014;11(22):49-64.

19. Koc RF, Leite MT, Hildebrandt LM, Linck CL, Terra MG, Gonçalves LTH. Depressão na percepção de idosas de grupos de convivência. Rev Enfermagem UFPE on line. 2013;7(9):5574-5582. doi: 10.5205/revol.3529-29105-1$\underline{S M .0709201327}$ 
20. Vieira KFL, Coutinho MPL, Saraiva ERA. A sexualidade na velhice: representações sociais de idosos frequentadores de um grupo de convivência. Psicologia Ciência e Profissão. 2016;36(1):196-209. doi: 101590/1982-3703002392013

21. Braga IB, Santana RC, Ferreira DMG. Depressão no Idoso. Id on Line Revista de Psicologia. 2015;9(26):142-151. 